\title{
Circular Polarization Modulation for Digital Communication Systems
}

\author{
Zain ul Abidin ${ }^{* 1}$, Pei Xiao ${ }^{* 2}$, Muhammad Amin ${ }^{3}$, Vincent Fusco ${ }^{4}$ \\ ${ }^{*}$ Centre for Communication Systems Research, University of Surrey, UK \\ ${ }^{3}$ Institute of Space Technology, Islamabad, Pakistan \\ ${ }^{4}$ Institute of ECIT, Queen's University Belfast \\ 1abidin_sheikh@yahoo.com, ${ }^{2}$ p.xiao@surrey.ac.uk, ${ }^{3}$ m.amin@ist.edu.pk, ${ }^{4}$ v.fusco@ecit.qub.ac.uk
}

\begin{abstract}
Conventional approaches of digital modulation schemes make use of amplitude, frequency and/or phase as modulation characteristic to transmit data. In this paper, we exploit circular polarization (CP) of the propagating electromagnetic carrier as modulation attribute which is a novel concept in digital communications. The requirement of antenna alignment to maximize received power is eliminated for $\mathrm{CP}$ signals and these are not affected by linearly polarized jamming signals. The work presents the concept of Circular Polarization Modulation for 2, 4 and 8 states of carrier and refers them as binary circular polarization modulation (BCPM), quaternary circular polarization modulation (QCPM) and 8-state circular polarization modulation $(8 \mathrm{CPM})$ respectively. Issues of modulation, demodulation, 3D symbol constellations and 3D propagating waveforms for the proposed modulation schemes are presented and analyzed in the presence of channel effects, and they are shown to have the same bit error performance in the presence of AWGN compared with conventional schemes while provide $3 \mathrm{~dB}$ gain in the flat Rayleigh fading channel.
\end{abstract}

\section{INTRODUCTION}

The concept of digital modulation by exploiting circular polarization is introduced in an active tagging system [1] in which an interrogator and a remote tag have a half duplex communication. Each of the orthogonal hands of circular polarization [2] represents an information bit as a modulation technique which simplifies demodulation process. The concept is extended to 4-state modulation in [3] where it is called CPQPSK. I and Q streams without being summed traditionally in a QPSK modulator are fed to a dual fed microstrip patch antenna [6] resonant at $7.6 \mathrm{GHz}$ to excite $\mathrm{CP}$ signals. As a fundamental requirement for the production of $\mathrm{CP}$ radiations, the feeds of the microstrip patch antenna need to be excited with sinusoids of equal magnitude of amplitude having a mutual phase difference of $\pi / 2$ as an essential requirement [4]. The individual ports of the antenna will excite horizontal and vertical components of the generated circularly polarized electromagnetic wave (i.e. $\mathbf{E}_{\mathrm{H}}$ and $\mathbf{E}_{\mathrm{V}}$ respectively) as a result of spatial combination in the antenna far field [7].

Let us define sinusoidal functions $\mathbf{E}_{\mathrm{IP}}, \mathbf{E}_{\mathrm{IN}}, \mathbf{E}_{\mathrm{QP}}$ and $\mathbf{E}_{\mathrm{QN}}$ as 'basis functions' for the $\mathrm{CP}$ modulation as follows

$$
\begin{gathered}
\mathbf{E}_{\mathrm{IP}}=\mathrm{E}_{\mathrm{o}} \cos \left(2 \pi f_{\mathrm{o}} \mathrm{t}\right) \\
\mathbf{E}_{\mathrm{IN}}=-\mathrm{E}_{\mathrm{o}} \cos \left(2 \pi f_{\mathrm{o}} \mathrm{t}\right) \\
\mathbf{E}_{\mathrm{QP}}=\mathrm{E}_{\mathrm{o}} \cos \left(2 \pi f_{\mathrm{o}} \mathrm{t}-\pi / 2\right) \\
\mathbf{E}_{\mathrm{QN}}=-\mathrm{E}_{\mathrm{o}} \cos \left(2 \pi f_{\mathrm{o}} \mathrm{t}-\pi / 2\right)
\end{gathered}
$$

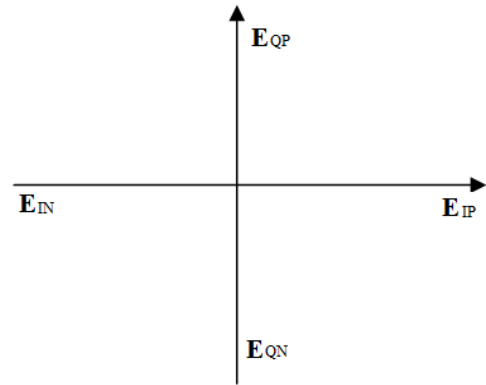

Figure 1. Rectangular coordinate representation of the basis functions of the CP modulation

The subscripts I and Q represent In-phase and Quadraturephase components, while $\mathrm{P}$ and $\mathrm{N}$ represent positive or negative polarity with amplitude $\mathrm{E}_{\mathrm{o}}$ and a frequency $f_{\mathrm{o}}$. A pair of signals from the defining functions of (1), when fed to a dual fed microstrip patch antenna, will generate a CP signal out of 8 possible states with a unique set of phase shift and sense of polarization that could be exploited for BCPM, QCPM and 8CPM. We represent the basis functions in rectangular coordinate system as shown in Figure 1 which will be used in our later discussion for $\mathrm{CP}$ symbol constellations. Here I and Q represent the horizontal and vertical electric field components of propagating carrier.

We now define 'Right hand Dot and Cross' notation to represent $\mathrm{CP}$ symbols in 3D-constellations where a dot corresponds to left hand $\mathrm{CP}$ or counter-clock-wise (CCW) sense and a cross represents right hand $\mathrm{CP}$ or clock-wise $(\mathrm{CW})$ sense. The CP symbol will be either outward or into the paper, indicated by the thumb of right hand while curling fingers in the direction of rotation described by the sense of $\mathrm{CP}$ as given in Table I. The phase angle of the generated CP signal will be the phase of axis coming first in this rotation.

TABLE I RIGHT HAND DOT AND CROSS NOTATION

\begin{tabular}{ccc}
\hline Sense of CP & Symbol Representation & Thumb Direction \\
\hline LHCP/CCW & Dot $\odot$ & Outward the paper \\
\hline RHCP $/ \mathrm{CW}$ & Cross $\otimes$ & Into the paper \\
\hline
\end{tabular}




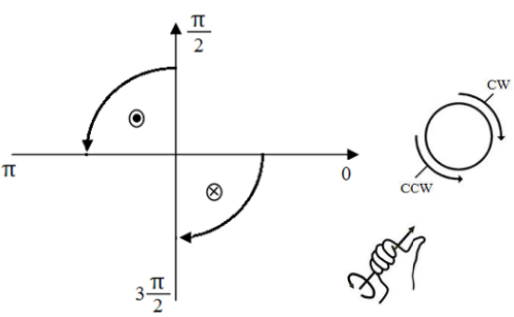

Figure 2. Determination of Phase in CP symbol constellation

As an example, the phase angles of two arbitrary CP symbols in second and fourth quadrant of rectangular coordinate system are $\frac{\pi}{2}$ and 0 radian with $\mathrm{CCW}$ and $\mathrm{CW}$ sense of $\mathrm{CP}$ with a symbol representation of a dot and a cross, respectively as illustrated in Figure 2.

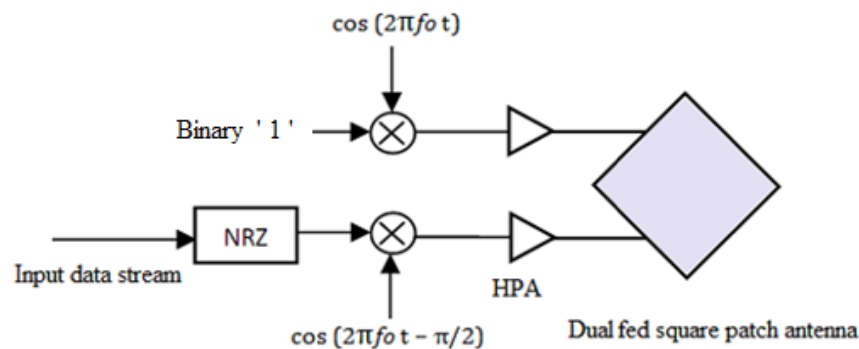

Figure 3. BCPM modulator

\section{BINARY CIRCULAR POLARIZATION MODULATION (BCPM)}

Figure 3 shows how the bi-state circular polarization modulation is implemented. It uses non-returning to zero, NRZ line coding scheme; to generate negative pulse for a bit ' 0 ' and a positive pulse for a bit ' 1 ', to feed one of the antenna ports while the other port is constantly fed with a positive level. The encoding scheme may be represented as Table II. Figure 3 shows that we have $\mathbf{E}_{I P}$ and $\mathbf{E}_{\mathrm{QN}}$ for a bit ' 0 ' and $\mathbf{E}_{\mathrm{IP}}$ and $\mathbf{E}_{\mathrm{QP}}$ for a bit ' 1 ' which after amplification by high power amplifier (HPA) produces RHCP and LHCP signal, respectively.

For test bits 01001 , the antenna feeds are plotted in Figure 4 which results in the generation of corresponding BCPM signals as shown in Figure 5. The BCPM symbol constellation is shown in Figure 6 in which orthogonal BCPM symbols are at $0^{\circ}$ as determined by Right hand dot and cross notation.

TABLE II BCPM SYMBOL ENCODING SCHEME

\begin{tabular}{cccc}
\hline Data bits & $\begin{array}{c}\text { Encoded CP } \\
\text { symbol }\end{array}$ & $\begin{array}{c}\text { Electric field } \\
\text { horizontal component }\end{array}$ & $\begin{array}{c}\text { Electric field } \\
\text { vertical component }\end{array}$ \\
\hline 0 & RHCP & $\mathrm{E}_{\mathrm{o}} \cos \left(2 \pi f_{\mathrm{o}} \mathrm{t}\right)$ & $-\mathrm{E}_{\mathrm{o}} \cos \left(2 \pi f_{\mathrm{o}} \mathrm{t}-\pi / 2\right)$ \\
\hline 1 & $\mathrm{LHCP}$ & $\mathrm{E}_{\mathrm{o}} \cos \left(2 \pi f_{\mathrm{o}} \mathrm{t}\right)$ & $\mathrm{E}_{\mathrm{o}} \cos \left(2 \pi f_{\mathrm{o}} \mathrm{t}-\pi / 2\right)$ \\
\hline
\end{tabular}

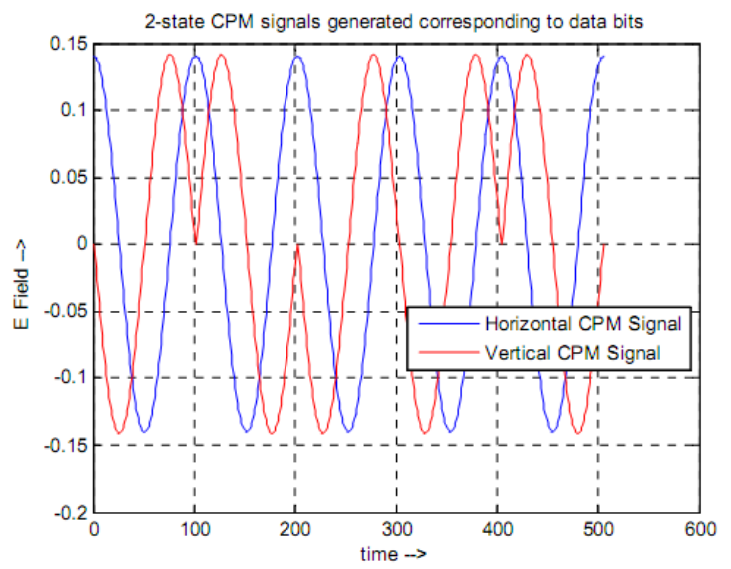

Figure 4. BCPM antenna feed signals for test bits 01001

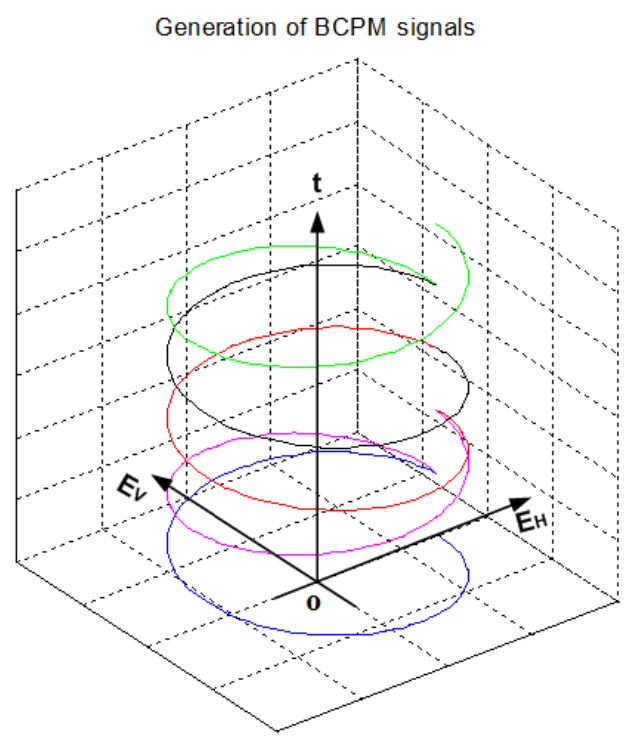

Figure 5. BCPM signal for test bits 01001

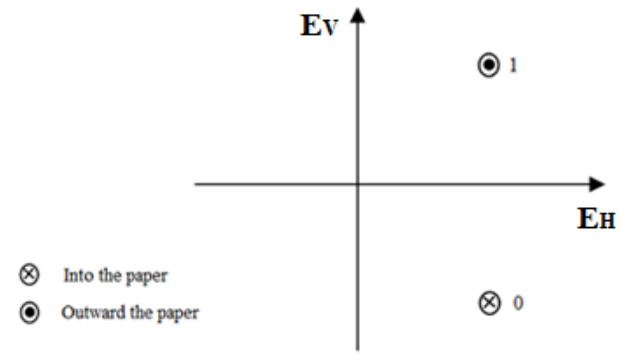

Figure 6. BCPM Symbol constellation 


\section{QUATERNERY CIRCULAR POLARIZATION MODULATION (QCPM)}

Table III illustrates the encoding scheme for QCPM which uses signals $\mathbf{E}_{\mathrm{IP}}$ and $\mathbf{E}_{\mathrm{IN}}$ as one of the feeds of the dual fed microstrip patch antenna, while $\mathbf{E}_{\mathrm{QP}}$ and $\mathbf{E}_{\mathrm{QN}}$ as the second feed with possible polarities providing 4 states of $\mathrm{CP}$ signal. The block diagram of QCPM modulator is given in Figure 7 in which incoming data bits are transformed into two parallel bit streams and then converted to bipolar signal level form using NRZ scheme and multiplied by orthogonal local oscillators (LO's) i.e. $\mathrm{E}_{0} \cos \left(2 \pi f_{0} \mathrm{t}\right)$ and $\mathrm{E}_{\mathrm{o}} \cos \left(2 \pi f_{0} \mathrm{t}-\pi / 2\right)$ in order to obtain required antenna feeds to generate a specific QCPM signal.

For test bits 00011011 , the antenna feeds are plotted in Figure 8 which results in the generation of corresponding four QCPM signals as shown in Figure 9. The 3D symbol constellation for QCPM can be given using the Right hand dot and cross notation which shows symbols for dibits ' 11 ' and ' 10 ' are at $0^{\circ}$ phase while for dibits ' 01 ' and ' 00 ' are at $180^{\circ}$ phase values as represented in Figure 10.

TABLE III QCPM SyMBOL ENCODING SCHEME

\begin{tabular}{cccc}
\hline Data bits & $\begin{array}{c}\text { Encoded CP } \\
\text { symbol }\end{array}$ & $\begin{array}{c}\text { Electric field } \\
\text { horizontal component }\end{array}$ & $\begin{array}{c}\text { Electric field } \\
\text { vertical component }\end{array}$ \\
\hline 00 & $180^{\circ}$ shifted LHCP & $-\mathrm{E}_{\mathrm{o}} \cos \left(2 \pi f_{\mathrm{o}} \mathrm{t}\right)$ & $-\mathrm{E}_{\mathrm{o}} \cos \left(2 \pi f_{\mathrm{o}} \mathrm{t}-\pi / 2\right)$ \\
\hline 01 & $180^{\circ}$ shifted RHCP & $-\mathrm{E}_{\mathrm{o}} \cos \left(2 \pi f_{\mathrm{o}} \mathrm{t}\right)$ & $\mathrm{E}_{\mathrm{o}} \cos \left(2 \pi f_{\mathrm{o}} \mathrm{t}-\pi / 2\right)$ \\
\hline 10 & RHCP & $\mathrm{E}_{\mathrm{o}} \cos \left(2 \pi f_{\mathrm{o}} \mathrm{t}\right)$ & $-\mathrm{E}_{\mathrm{o}} \cos \left(2 \pi f_{\mathrm{o}} \mathrm{t}-\pi / 2\right)$ \\
\hline 11 & LHCP & $\mathrm{E}_{\mathrm{o}} \cos \left(2 \pi f_{\mathrm{o}} \mathrm{t}\right)$ & $\mathrm{E}_{\mathrm{o}} \cos \left(2 \pi f_{\mathrm{o}} \mathrm{t}-\pi / 2\right)$ \\
\hline
\end{tabular}

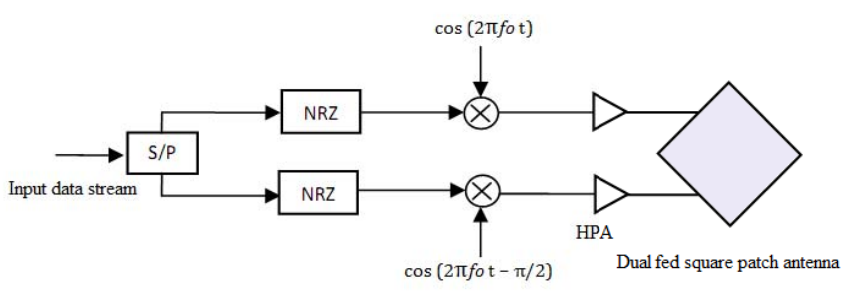

Figure 7. QCPM Modulator

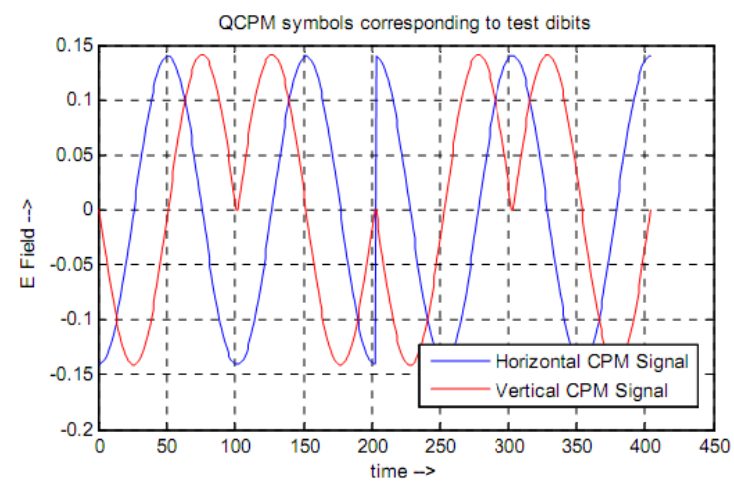

Figure 8. QCPM antenna feed signals for test bits 00011011

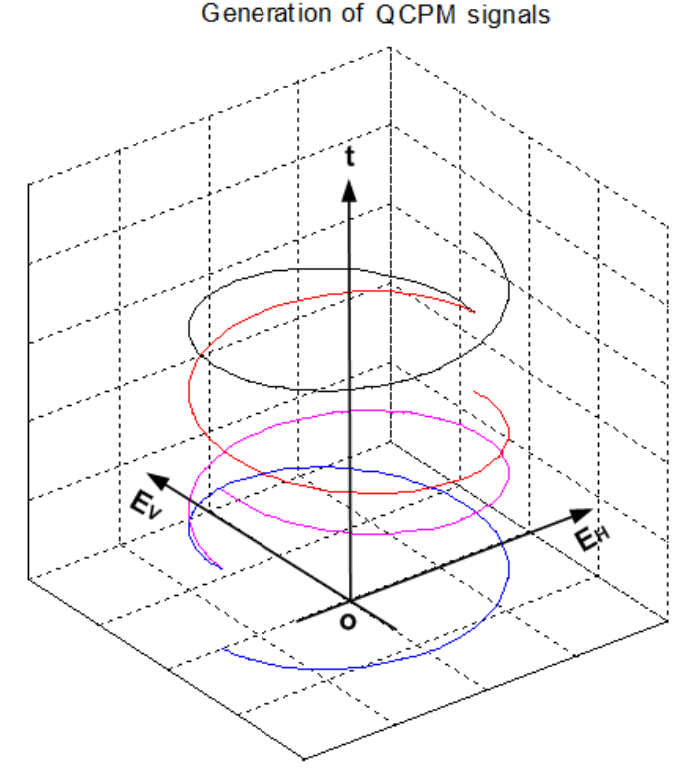

Figure 9. QCPM signal for test bits 00011011

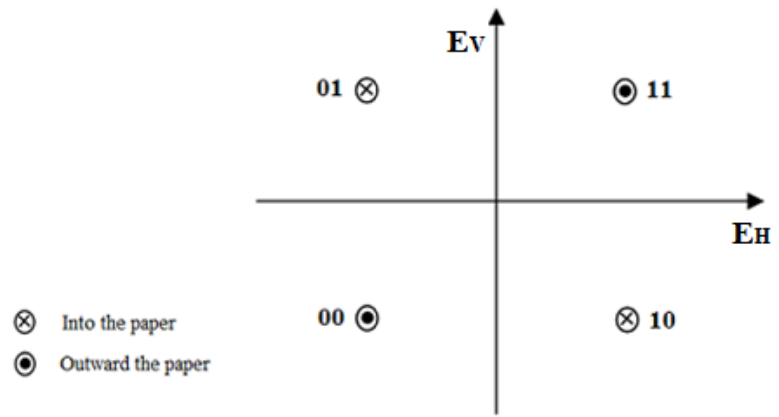

Figure 10. QCPM Symbol Constellation

\section{Eightstate CiRcular Polarization Modulation} (8CPM)

The encoding of incoming tribits to respective $8 \mathrm{CPM}$ symbols can be performed as shown in Table IV where the phase angle of $8 \mathrm{CPM}$ signal will be $0^{\circ}$ for tribits ' $010^{\prime}$ ' and ' 011 '; $180^{\circ}$ for ' $000^{\prime}$ ' and ' 001 '; $90^{\circ}$ for ' 101 ' and ' 111 '; $270^{\circ}$ for ' 100 ' and ' 110 '. Figure 12 shows how the scheme can be implemented. Depending upon the MSB of the incoming tribit, the rest of the two bits would be directed to the upper or lower block of the modulator to have respective antenna feeds to generate the required $8 \mathrm{CPM}$ signal.

For test bits $000001010011100101110 \quad 111$, the antenna feeds are plotted in Figure 12 which results in the generation of corresponding 8 QCPM signals as shown in Figure 13. Figure 14 shows 8CPM symbol constellation. 


\begin{tabular}{cccc}
\hline Data bits & $\begin{array}{c}\text { Encoded CP } \\
\text { symbol }\end{array}$ & $\begin{array}{c}\text { Electric field } \\
\text { horizontal component }\end{array}$ & $\begin{array}{c}\text { Electric field } \\
\text { vertical component }\end{array}$ \\
\hline 000 & $180^{0}$ shifted LHCP & $-\mathrm{E}_{\mathrm{o}} \cos \left(2 \pi f_{\mathrm{o}} \mathrm{t}\right)$ & $-\mathrm{E}_{\mathrm{o}} \cos \left(2 \pi f_{\mathrm{o}} \mathrm{t}-\pi / 2\right)$ \\
\hline 001 & $180^{0}$ shifted RHCP & $-\mathrm{E}_{\mathrm{o}} \cos \left(2 \pi f_{\mathrm{o}} \mathrm{t}\right)$ & $\mathrm{E}_{\mathrm{o}} \cos \left(2 \pi f_{\mathrm{o}} \mathrm{t}-\pi / 2\right)$ \\
\hline 010 & RHCP & $\mathrm{E}_{\mathrm{o}} \cos \left(2 \pi f_{\mathrm{o}} \mathrm{t}\right)$ & $-\mathrm{E}_{\mathrm{o}} \cos \left(2 \pi f_{\mathrm{o}} \mathrm{t}-\pi / 2\right)$ \\
\hline 011 & LHCP & $\mathrm{E}_{\mathrm{o}} \cos \left(2 \pi f_{\mathrm{o}} \mathrm{t}\right)$ & $\mathrm{E}_{\mathrm{o}} \cos \left(2 \pi f_{\mathrm{o}} \mathrm{t}-\pi / 2\right)$ \\
\hline 100 & $270^{0}$ shifted RHCP & $-\mathrm{E}_{\mathrm{o}} \cos \left(2 \pi f_{\mathrm{o}} \mathrm{t}-\pi / 2\right)$ & $-\mathrm{E}_{\mathrm{o}} \cos \left(2 \pi f_{\mathrm{o}} \mathrm{t}\right)$ \\
\hline 101 & $90^{0}$ shifted LHCP & $-\mathrm{E}_{\mathrm{o}} \cos \left(2 \pi f_{\mathrm{o}} \mathrm{t}-\pi / 2\right)$ & $\mathrm{E}_{\mathrm{o}} \cos \left(2 \pi f_{\mathrm{o}} \mathrm{t}\right)$ \\
\hline 110 & $270^{0}$ shifted LHCP & $\mathrm{E}_{\mathrm{o}} \cos \left(2 \pi f_{\mathrm{o}} \mathrm{t}-\pi / 2\right)$ & $-\mathrm{E}_{\mathrm{o}} \cos \left(2 \pi f_{\mathrm{o}} \mathrm{t}\right)$ \\
\hline 111 & $90^{0}$ shifted RHCP & $\mathrm{E}_{\mathrm{o}} \cos \left(2 \pi f_{\mathrm{o}} \mathrm{t}-\pi / 2\right)$ & $\mathrm{E}_{\mathrm{o}} \cos \left(2 \pi f_{\mathrm{o}} \mathrm{t}\right)$ \\
\hline
\end{tabular}

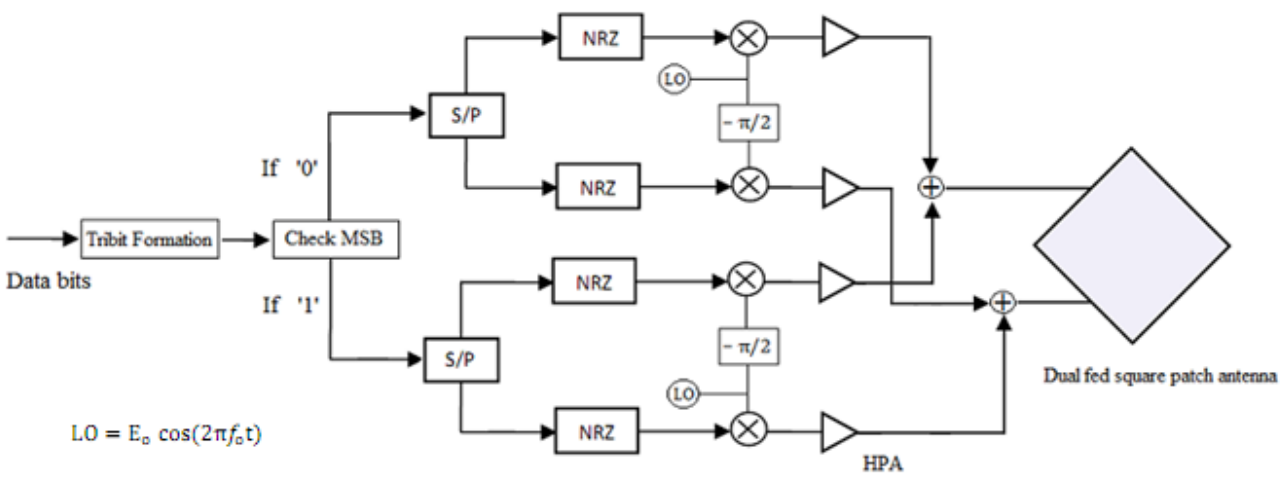

Figure 11. 8CPM modulator

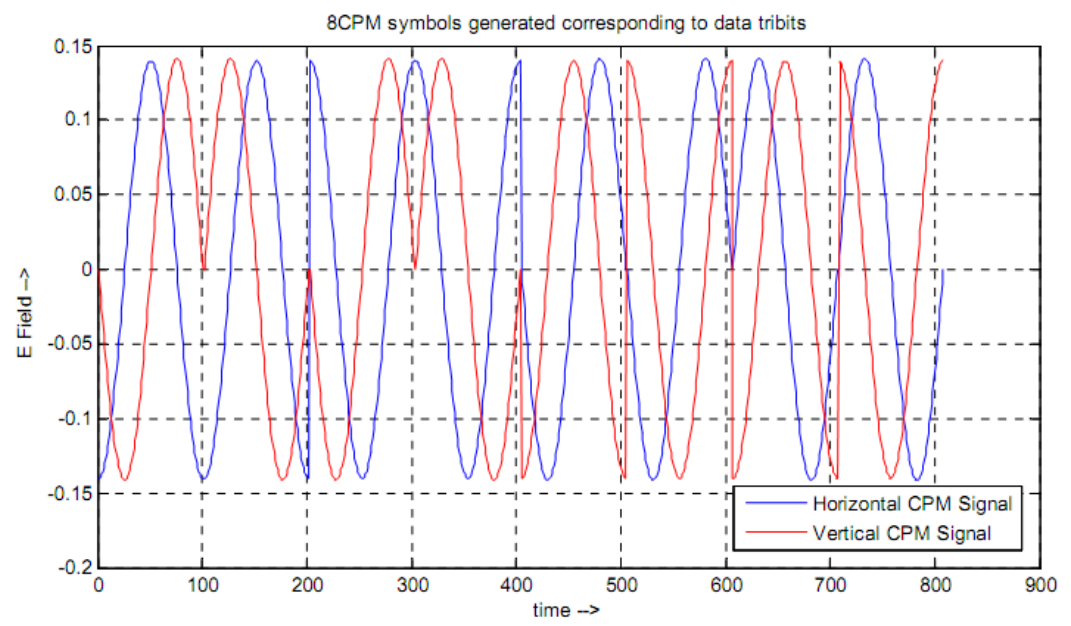

Figure 12. 8CPM antenna feed signals 8 possible test tribits 


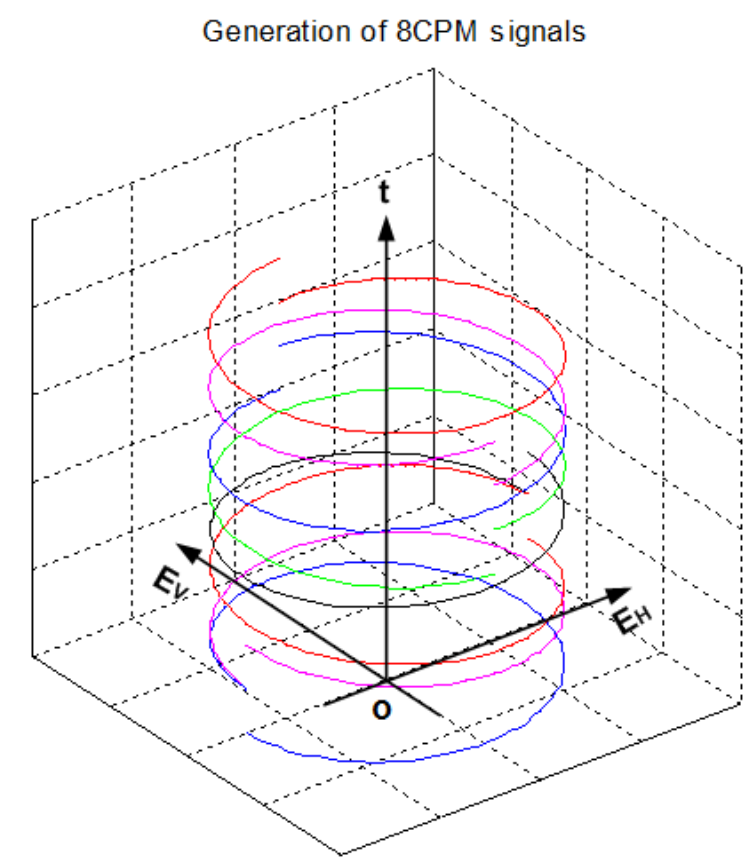

Figure 13. 8CPM signal for 8 possible test tribits

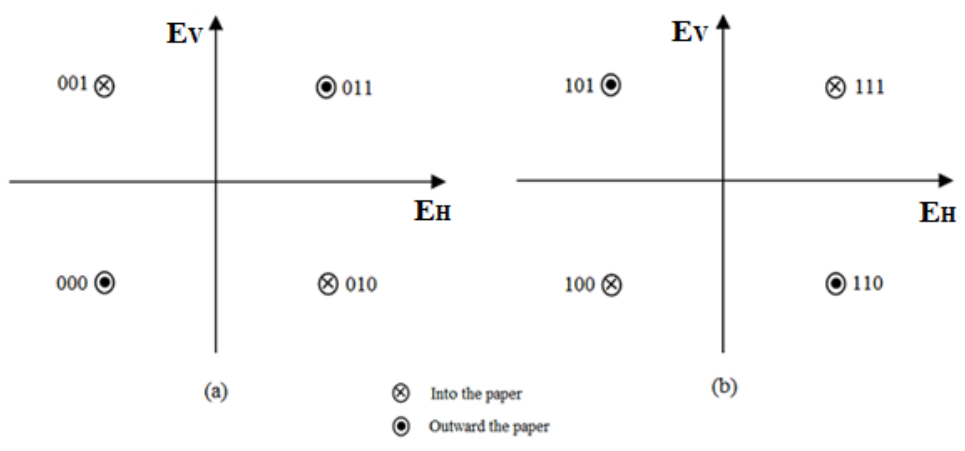

Figure 14. 8CPM symbol Constellation

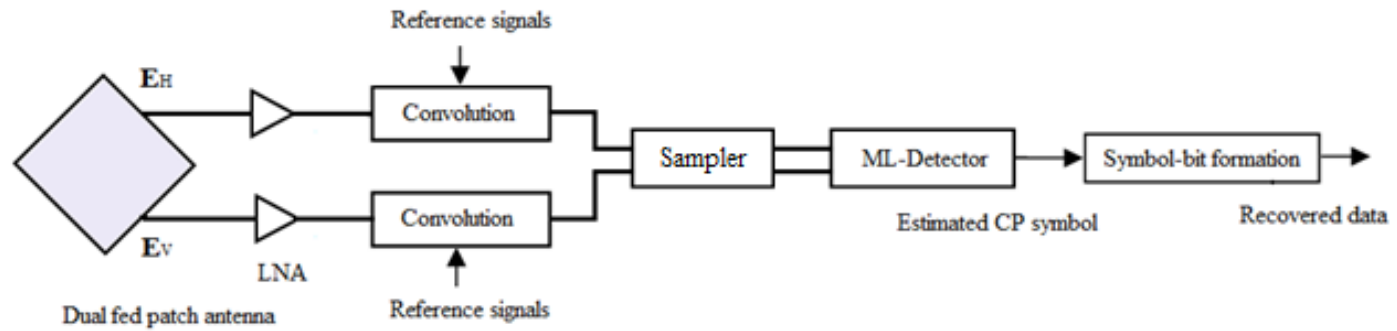

Figure 15. Demodulation scheme for CP modulation
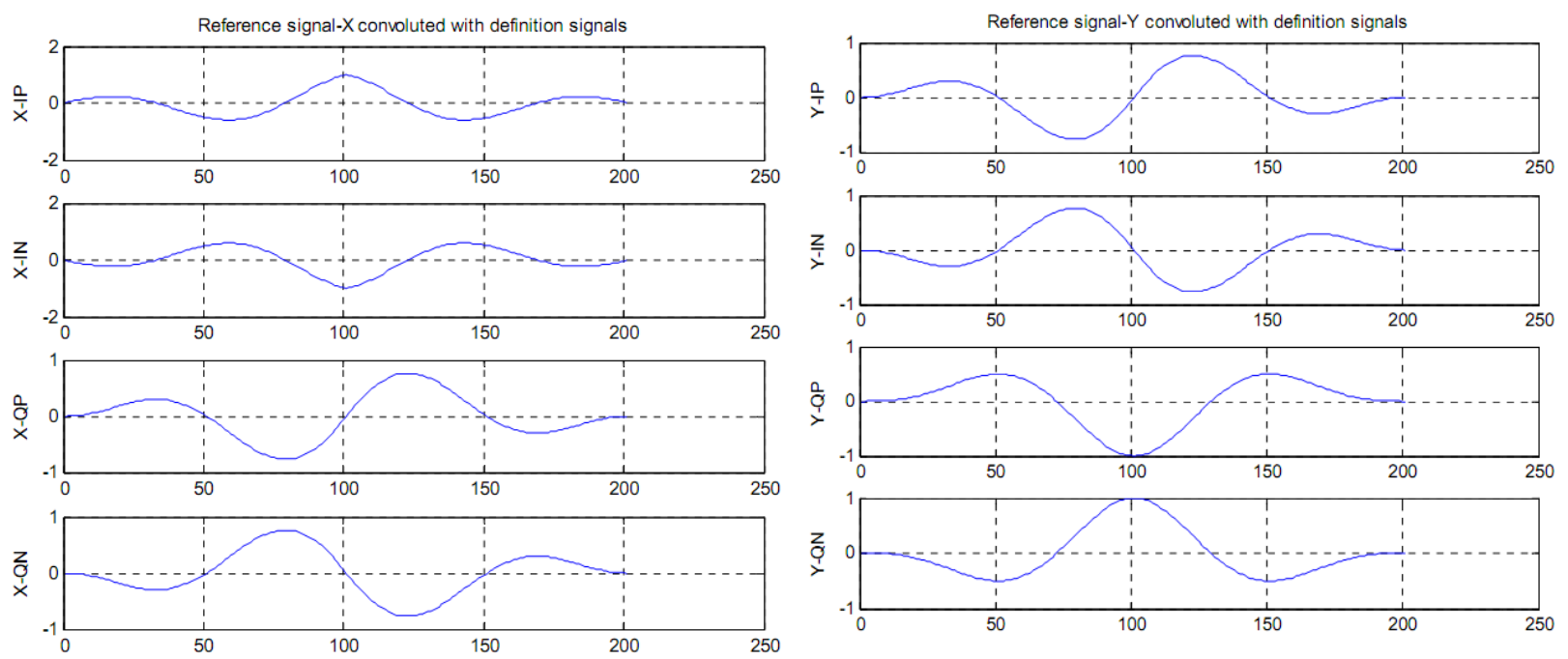

Figure 16. Reference signal $\mathrm{X}$ convolved with the basis signals

Figure 17. Reference signal $\mathrm{Y}$ convolved with the basis signals 


\section{DEMODULATION OF CP SIGNALS}

The reciprocity of a dual fed microstrip patch antenna enables us to regenerate horizontal and vertical components $\boldsymbol{E}_{H}$ and $\boldsymbol{E}_{V}$ respectively of received EM radiation for demodulation purpose. As depicted in Figure 15, these electric field components after amplification by a low noise amplifier (LNA) are convolved with reference signals, $\boldsymbol{E}_{x}$ and $\boldsymbol{E}_{y}$ such that $\boldsymbol{E}_{x}=\boldsymbol{E}_{\mathrm{IP}}$ and $\boldsymbol{E}_{y}=\boldsymbol{E}_{\mathrm{QP}}$. Figure 16 and 17 show continuous time signals obtained by the convolution of reference signals, i.e. $\mathrm{X}$ and $\mathrm{Y}$ with the defining signals respectively. The magnitude of central samples (say at 100 in Figs. 16 and 17) in the convolved waveform can act as signature in detection process. These two sets of quadsignatures are used by ML detector for estimation of data transmitted by seeking these signatures out of received signal to estimate the transmitted $\mathrm{CP}$ symbol based upon suitable thresholds in case of BCPM, QCPM or $8 \mathrm{CPM}$.

\section{ANALYSIS IN THE PRESENCE OF CHANNEL EFFECTS}

Matlab simulations of standard Monte Carlo technique [5] for the performance evaluation in terms of Bit Error Rate (BER) vs. Signal to noise ratio (SNR) were performed and compared with conventional Phase Shift Keying (PSK) scheme. The probability of bit error for BCPM in Additive White Gaussian channel (AWGN) was found close to the conventional BPSK. The simulations for bit error performance evaluation of BCPM in flat fading (FF) Rayleigh channel shows $3 \mathrm{~dB}$ gain compared with BPSK due to inherent diversity of circular polarization as depicted by the plot of Figure 18.

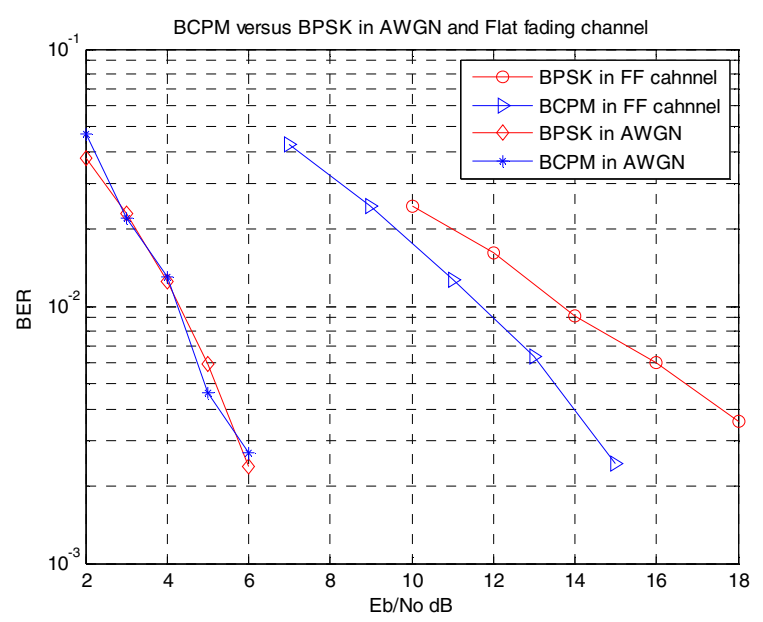

Figure 18. Analysis of BCPM in AWGN and Flat fading channel

\section{CONCLUSION}

The paper presents a novel concept in the field of digital communication by exploiting circular polarization as a characteristic to modulate data. This is different from conventional approaches that modulate data using amplitude, frequency or phase of electromagnetic carrier as modulation attributes. Circular Polarization Modulations for 2, 4 and 8 states have been presented with thorough concept of symbol encoding, constellation, modulation, demodulation and the waveforms of the propagating carrier. The proposed $\mathrm{CP}$ modulation techniques provide inherent benefits of circular polarization as well as diversity gain in fading channels.

\section{REFERENCES}

[1] Marcel A. Kossel, Roland Küng, Hansruedi Benedickter and Werner Bächtold, "An Active tagging system using Circular-Polarization modulation", IEEE Transactions on Microwave Theory and Techniques, vol. 47, no. 12, pp. 2242-2248, December 1999.

[2] B. Y. Toh and R. Cahill and V. F. Fusco, "Understanding and measuring Circular polarization”, IEEE Transactions on Education, vol. 46, no. 3 pp. 313-318, August 2003.

[3] M. Amin, S. Ahmed, V. Fusco, H. Cantu and T. Ratnarajah, "The effect of spatial axial ratio variation on QPSK modulation encoded using orthogonal circularly polarized signals", Proceedings of the 10th European Conference on Wireless Technology, Munich Germany, October 2007.

[4] Constantine A. Balanis, Antenna Theory, Analysis and Design, Third Edition, 047166782X, Wiley-Interscience, 2005.

[5] Bernard Sklar, Digital Communications, Fundamentals and Applications, Second Edition, ISBN 0130847887, Prentice Hall International, 2001.

[6] David M. Pozar, "Microwave Engineering", Third Edition, ISBN: 0471448788, Wiley, 2005.

[7] IEEE Standard Definitions of terms for Antennas, IEEE Std, 145-1983. 